\title{
Bio-inspired Two Target Resolution at Radio Frequencies
}

\author{
Krasin Georgiev, Alessio Balleri \\ Centre for Electronic Warfare, Information and Cyber \\ Cranfield University \\ Defence Academy of UK, Shrivenham, SN6 8LA, UK \\ k.georgiev@cranfield.ac.uk, a.balleri@cranfield.ac.uk
}

\author{
Andy Stove \\ Stove Specialties \\ Hove, E. Sussex BN3 5NL, UK \\ andystove@compuserve.com
}

\author{
Marc Holderied \\ School of Biological Sciences \\ Bristol University \\ Bristol, BS8 1TQ, UK \\ Marc.Holderied@bristol.ac.uk
}

\begin{abstract}
Echolocating bats show a unique ability to detect, resolve and discriminate targets. The Spectrogram Correlation and Transformation (SCAT) receiver is a model of the Eptesicus fuscus auditory system that presents key signal processing differences compared to radar which may offer useful lessons for improvement. A baseband version of the SCAT is used to investigate advantages and disadvantages of bat-like signal processing against the task of target resolution. The baseband receiver is applied to $R F$ experimental data and results show higher range resolution than the reciprocal of the transmitted bandwidth can be achieved for two closely spaced scatterers.
\end{abstract}

\section{INTRODUCTION}

Detection and recognition of a target amongst other interfering objects has been both a theoretical and a practical problem since the early ages of radar systems [1]. One of the first challenges in this area was robust resolution of individual target scatterers in complex clutter environments and the subject has now advanced to the latest attempts to develop cognitive sensing capabilities [2], [3].

Bats can use echolocation alone to detect, localise and discriminate both moving and stationary targets in a complex environment [4], [5]. A key and interesting feature of bat echolocation is that recent behavioral experiments have demonstrated bats are able to resolve closely spaced scatterers. A behavioural experiment presented in [6] and [7] showed that the big brown bat (Eptesicus fuscus) could achieve a two-point resolution between 2 and $10 \mu$ s with a transmitted waveform bandwidth of $85 \mathrm{kHz}$. Similarly, the bat Megaderma lyra was able to discriminate between two phantom target echoes delayed of about $1 \mu \mathrm{s}$ by transmitting waveforms with a bandwidth up to $100 \mathrm{kHz}$ [8]. Discrimination of surface structures was demonstrated in [9] where a few Glossophaga soricina individuals were trained to distinguish between smooth and coarse surfaces with a structure depth difference greater than $0.38 \mathrm{~mm}$. In these experiments, the bats achieved good discrimination performance by transmitting frequency modulated down-chirp signals consisting of three harmonics covering the three frequency bandwidths from $95 \mathrm{kHz}$ to $55 \mathrm{kHz}$, from 150 $\mathrm{kHz}$ to $86 \mathrm{kHz}$ and from $190 \mathrm{kHz}$ to $140 \mathrm{kHz}$, respectively. The experiments above have clearly shown that certain bat species can achieve higher range resolution than the one over the signal bandwidth limit achieved by a typical radar matched filter.

Krasin Georgiev's work was supported by Cranfield Defence and Security under the $\mathrm{CDS} \mathrm{PhD}$ bursary scheme.
There is biological evidence that indicates that the way bats process signals in the receiving auditory system is not equivalent to the matched filter used in radar and sonar systems, and hence it can be useful to investigate differences and similarities and study how these can be used to improve the performance of synthetic sensors. In the bat auditory system, according to the existing literature, both the emitted call and the received echo are passed through an auditory periphery followed by some higher level brain structures. The auditory periphery includes the outer, the middle and the inner ear of the bat and is modelled as a bank of filters that provides a time-frequency representation of the input signal (the so called auditory spectrogram) [10], [11], [12]. The central frequencies of each filter in the bank are such that the signal frequency content is sampled in a non-linear scale and typically they follow a hyperbolic or a logarithmic curve. The following central nervous system processing provides an estimate of the time delay between the call and the echo and provides cues about the structure of each echo.

Multiple studies have proposed a variety of models of the bat auditory system and explored their performance through simulations and experiments with ultrasound [10]-[17]. The Spectrogram Correlation and Transformation (SCAT) receiver is one of these models and was proposed by Saillant et al. in [10] for the bat Eptesicus fuscus. The SCAT consists of three processing modules: the cochlear block, the temporal block (spectrogram correlation) and the spectral block (spectrogram transformation).

The model considers down-chirp signals in transmission. The cochlear block produces an auditory spectrogram of a target echo which is then converted into the target range profile in the temporal block. The emitted down-chirp and the received echo are passed through the bank of bandpass filters and each filter output is then rectified, low-pass filtered and non-linearly transformed within the cochlear processing. In the temporal block, the output of each filter channel is delayed separately according to the design of the emitted chirp to ensure that the reflections from the same scatterer are aligned in the time domain between channels. Summing the outputs of all frequency channels provides a rough range profile of the target to achieve a kind of pulse compression. The main targets can be identified by peak localisation (temporal processing).

The spectrogram block is responsible for extracting the fine range characteristics of a target echo and it can be summarised as follows. For each target the energy spectrum is calculated 
for the central frequencies of the bandpass filters. Each filter output is integrated over a specific time interval around the target. Then it is normalised by the energy of the emitted signal for the corresponding filter (it varies by filter if the chirp is not linear). This is the frequency representation of the spectral output. The fine delay profile is calculated as the weighted inverse cosine transform of the normalised energy spectrum. The weights are introduced to compensate for the hyperbolic shape of the chirp. Calculations involve the removal of the mean energy as explained in [18]. This is the time representation of the spectral output.

The SCAT receiver performance was studied analytically in [19] and [18] where the outputs of the bandpass filters representing the auditory periphery were approximated with cosines shaped by Gaussian envelopes. Peremans and Hallam [19] showed the limitations of the temporal block. Park and Allen [18] further explored the output of the spectral block.

There has been very little research to investigate the performance of these models with radar signals. The Baseband Spectrogram Correlation and Transformation Receiver (BSCT) is a receiver that was developed based on the SCAT model to allow a mathematical treatment of the output of the SCAT and the processing of RF signals centred on very high carrier frequencies [20]. To date, the BSCT has been used to study the output of two closely-spaced scatterers both analytically and experimentally at ultrasound frequencies [21].

In this paper, we assess the performance of BSCT against radio frequency (RF) measurements. The response of two closely spaced targets was measured using a Vector Network Analyser (VNA) transmitting a stepped frequency waveform. The experiment tested the range resolution properties for two stationary targets as a function of the transmitted signal bandwidth. By increasingly reducing the bandwidth of the transmitted signal we compared the performance of the BSCT with respect to the Matched Filter (MF). Two flat plates were used as a physical realisation of two scatterers.

\section{BIO-INSPIRED MODEL DESCRIPTION}

The BSCT was developed to allow an analytical treatment of the spectral output of the SCAT [20]. A block digram of the BSCT is shown in Fig.1. The input signal is passed through a bank of filters, all characterised by the same design but centred at different carrier frequencies. The amplitude of the output of each filter is then used to calculate the energy of each channel. An inverse Fourier transform of the sequence of energy samples is then used to obtain the time-domain output of the block. It has been shown that, when the input of the model consists of the echoes from two closely spaced scatterers, the BSCT output is a profile that provides information on the relative distance between the two scatters and that discards the information on the scatter absolute locations. The output is also referred to as the fine delay profile in the SCAT literature [18], [19].

The case of echoes from two closely spaced targets for a linear chirp in transmission is treated in [21] for a bank of flat filters with a constant spacing so to allow the use of a conventional Inverse Fast Fourier Transform (IFFT). The core of the algorithm, in this case, is equivalent to firstly calculating the frequency profile of the target with the following steps:

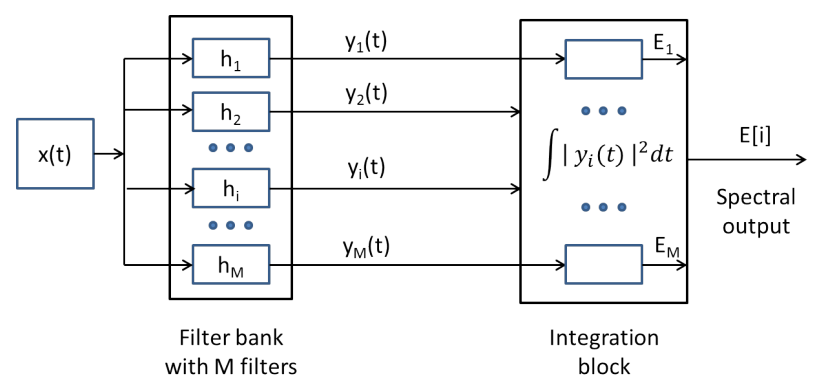

Fig. 1. Baseband implementation of SCAT spectral processing [20]. Model input $x(t)$ is analytical signal. It is passed through filter bank of $\mathbf{M}$ complex bandpass filters $h_{i}$. The absolute value of each filter output $y_{i}$ is squared and integrated over time to get the energy $E[i]$ of the corresponding frequency.

- calculate the energy density spectrum of the return signal $x(t)$

$$
P(f)=|X(f)|^{2}
$$

- $\quad$ average as

$$
E[i]=\frac{1}{B} \int_{f_{i}-B / 2}^{f_{i}+B / 2} P(f) d f
$$

so that the signal spectral energy of the input signal is integrated for the $\mathrm{i}$-th channel over a bandwidth $B$ around the selected centre frequencies $f_{i}$.

and then take the inverse Fourier transform of the frequency profile E[i] to obtain the time/space domain output

For two ideal scatterers the energy spectral density can be expressed as

$$
P(f)=2 P_{C}(f)\left[\cos \left(2 \pi f \tau+\psi_{\tau}\right)+1\right]
$$

where $\tau$ is the delay between the two targets, $\psi_{\tau}=$ $2 \pi \tau f_{0}$ is a phase component resulting from frequency downconversion and $P_{C}(f)$ is the energy spectrum of the transmitted call. It can be shown that Eq.(1) becomes

$$
E[i]=2 E_{C}\left[\operatorname{sinc}(\tau B) \cos \left(2 \pi \tau f_{i}+\psi_{\tau}\right)+1\right]
$$

where $E_{C}$ is a constant and $\operatorname{sinc}(x)=\sin (\pi x) /(\pi x)$ defines a sinc function.

The profile in (3) can be used directly to identify the spacing between the two scatterers. Removing the constant component in (3) will result in a single sinusoid which, in the time domain, translates into two sinc functions centred at $\pm \tau$. A simple solution to remove the constant component is to subtract the mean of the frequency profile $E[i]$ as

$$
E_{a c}[i]=E[i]-\frac{1}{B_{C}} \sum_{i=1}^{N} E[i]
$$

Unlike the matched filter response, the output of the BSCT encodes directly the spacing between the scatterers and not the absolute range of the targets. The problem of measuring the relative distance is converted to a problem of measuring the range to a single target. As the representation is symmetrical, there is a second peak at negative delays but the peaks interfere less than the peaks produced by a matched filter because the separation is double. 
We will further refer to the fine delay profile as the spacing profile to emphasise the nature of the information conveyed, i.e. not the absolute location of the scatterers but the spacing between them.

For practical applications, in the presence of clutter and multiple scatterers, the echo has to be firstly compressed in order to identify and select the range bins around the two interfering targets. This way, the noise level is reduced and the scatterers from other targets are not processed.

\section{DESCRIPTION OF THE EXPERIMENTS}

Measurements were performed at radio frequency using two $5 \times 4 \mathrm{~cm}$ horn antennas and a Vector Network Analyzer (VNA) (MS46322A, Anritsu) to transmit a stepped frequency waveform between $13 \mathrm{GHz}$ to $17 \mathrm{GHz}$ with a step frequency of $250 \mathrm{kHz}$. The VNA provides a measurement of the frequency response of a target so that High Range Resolution Profiles (HRRPs) can be obtained by taking an Inverse Fast Fourier Transform (IFFT) of the output data. The use of the VNA allowed us to vary the range resolution of the data, as desired, by processing different sub-bands within the available $4 \mathrm{GHz}$ bandwidth.

Two closely spaced flat plates (Fig. 2) were measured to investigate the range resolution properties of the BSCT model. During the measurements, the targets were facing the transmitting and receiving antennas with an aspect angle that provided approximately equal Radar Cross Sections (RCS). The radial distance between the two flat plates was kept constant at $0.12 \mathrm{~m}$ and both the targets and the antennas were placed $1.1 \mathrm{~m}$ above the floor level. The distance between the antennas and the closer flat plate was about $3.1 \mathrm{~m}$.

The BSCT algorithm presented in [21] was modified to account for the type of input provided by the VNA, i.e the frequency response of the targets.

The BSCT frequency profile was obtained with the following steps:

1) isolate the range bin of the target $\left[x_{1}, x_{2}\right], \mathrm{m}$ by Inverse FFT of the measured frequency response $X_{M}(f)$ and zeroing all elements outside the interval $t_{1}=2 x_{1} / c, \mathrm{~s}$ and $t_{2}=2 x_{2} / c, \mathrm{~s}$ in the time representation of the signal, where $c$ is the speed of light, $x_{1}=1 \mathrm{~m}$ and $x_{2}=5 \mathrm{~m}$. Apply FFT to get the frequency response of the target.

2) calculate the energy spectrum by multiplying the target frequency response $X_{M}(f)$ by its complex conjugate;

3) average the spectrum over a bandwidth $B$ for $K$ central frequencies linearly spaced in the measurement band. Averaging was performed over 100 samples and the signal was downsampled by 100 which resulted in $B=25 \mathrm{MHz}$ and $K$ between 160 for bandwidth $4 \mathrm{GHz}$ and 8 for $0.2 \mathrm{GHz}$.

The BSCT spacing profile was then obtained by:

1) modify the frequency profile by subtracting its average; this will result in a real signal with both positive and negative values.

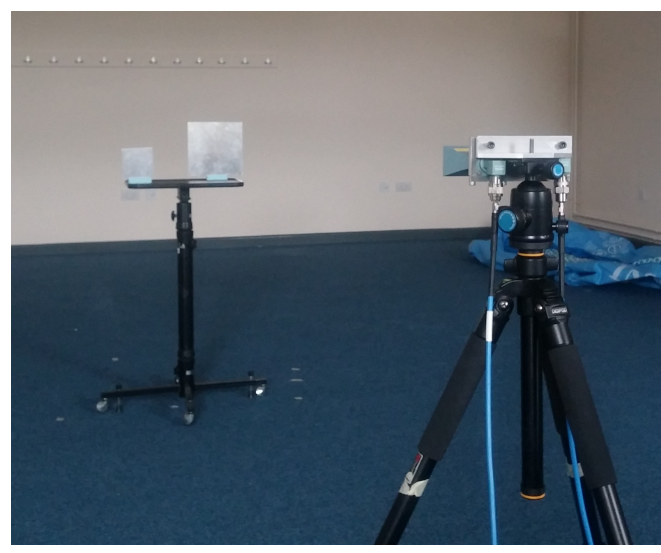

Fig. 2. Two flat plates as a target

2) inverse Fourier transform with an IFFT the modified frequency profile; zero-padding was applied to achieve an appropriate time sampling rate.

Targets were identified by peak extraction. A threshold equal to the mean signal power plus two times the standard deviation of the data was used. The minimal peak prominence to consider it as a separate peak was $3 \mathrm{~dB}$. Target spacing was estimated as the distance between the two highest peaks in the MF range profile and as the location of highest peak in the BSCT spacing profile.

\section{RESULTS}

The target range profiles of the two flat plates were studied using both the MF and the BSCT as a function of the range resolution.

For the analysis of the results, the classical range resolution was defined as the reciprocal of the signal bandwidth transformed into a distance:

$$
d_{R}=\frac{1}{B_{C}} \frac{c}{2}
$$

The spacing between the two targets $d$ was expressed relative to the classical resolution limit $d_{R}$ as the relative spacing $\bar{d}=d / d_{R}$. The relative spacing is a parameter that embeds the information about the bandwidth of the transmitted signal and hence allows to encode the resolution performance with a single parameter.

Scatterers spaced well above the classical resolution limit (5), e.g. $\bar{d} \geq 2$, for good signal to noise ratio (SNR) leads to spacing (range) profile as in Fig. 3a (Fig. 3c). There is a strong peak at the true target spacing. In the example provided, the signal bandwidth $B_{C}$ was $4 \mathrm{GHz}$ and the relative spacing was $\bar{d}=3.28$. The continuous vertical line marks the true scatterer spacing and the dashed line is at the classical resolution limit. The red circle marks the estimate of the spacing based on the peak location for the BSCT and the estimated range of the scatters for the MF. Another peak close to the resolution limit can be observed in Fig. $3 \mathrm{a}$ and this is likely due to residual background clutter as it cannot be reproduced with simulations. 


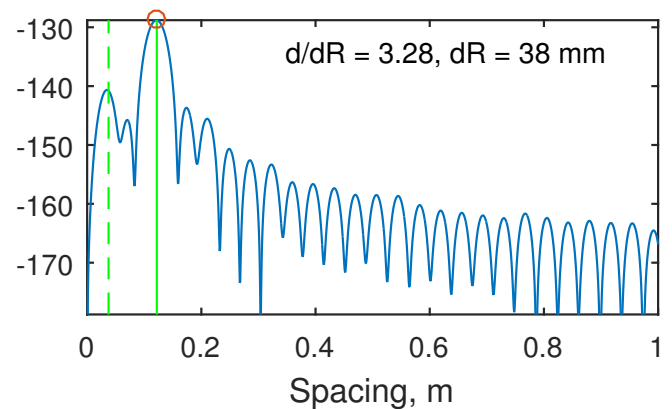

(a) BSCT, Signal bandwidth $4 \mathrm{GHz}$

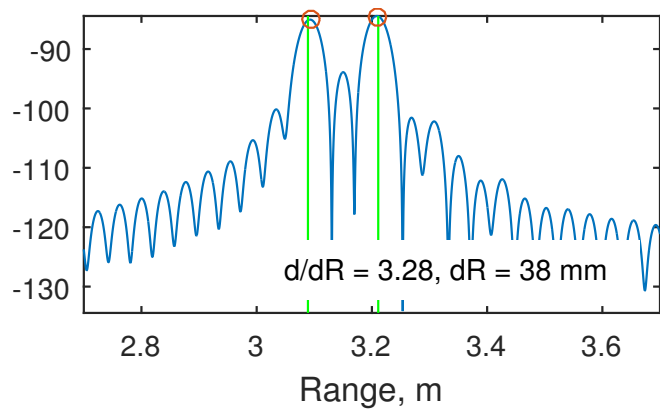

(c) MF, Signal bandwidth $4 \mathrm{GHz}$

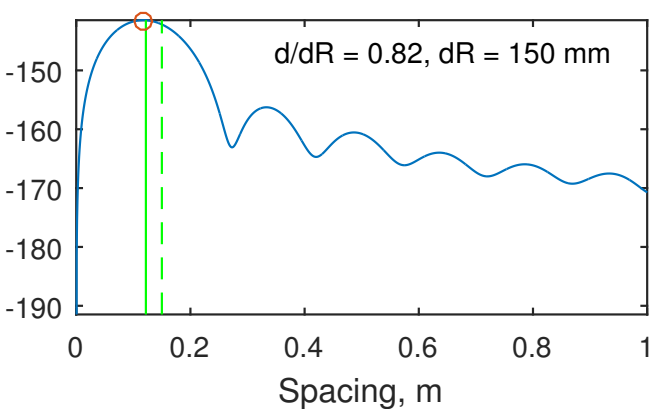

(b) BSCT, Signal bandwidth $0.5 \mathrm{GHz}$

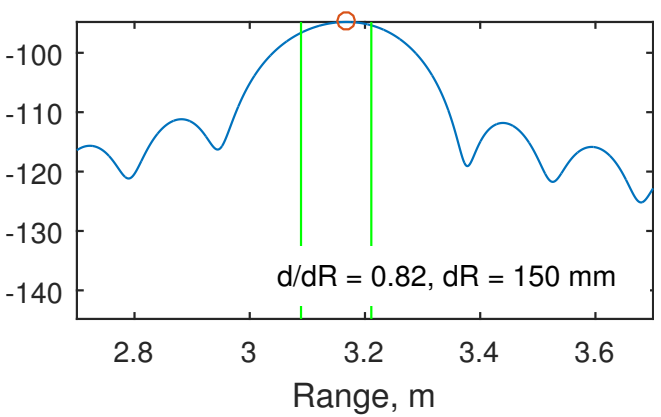

(d) MF, Signal bandwidth $0.5 \mathrm{GHz}$

Fig. 3. BSCT spacing profile (top) and regular range profile (bottom) for target spacing $12.2 \mathrm{~cm}$. The bandwidth is $4 \mathrm{GHz}$ (left) and $0.5 \mathrm{GHz}$ (right). The true target spacing or range is marked by continuous vertical line. The dashed line denotes the resolution limit. The peak of the profile is marked by a circle.

We would expect the target separation to become less clear when the resolution is reduced by reducing the signal bandwidth. Data to investigate this was obtained by taking a $0.5 \mathrm{GHz}$ section of the whole $4 \mathrm{GHz}$ data set. The interference pattern for scatterer spacing close to the resolution limit (5), e.g. $0.5<\bar{d}<2$, is shown in Fig. 3b and Fig. 3d. Results clearly show that the BSCT provides the correct spacing between the two scatterers whilst the two MF peaks have merged.

Target spacing estimates based on the BSCT and the conventional MF (or just IFFT in our case) are presented as a function of the relative spacing $\bar{d}$ in Fig. 4. In the figure, the BSCT target estimates are presented as circles and the MF based estimates, when available, are marked as crosses. The dotted line is the classical resolution limit $d_{R}$. At low bandwidths the target interaction peak tends to merge with the classical resolution limit for the BSCT. For the MF case both scatterer peaks merge. It can be seen that targets can be resolved at $0.7 d_{R}$ and above (Fig. 4) for the spacing model (BSCT) and at above $1.5 d_{R}$ for the range model (MF).

The resolution capabilities are influenced by the central frequency of the signal. We were able to examine this effect by taking different lower-resolution sub-sections of the total bandwidth over which we have collected data (Fig. 5) and produce different estimates of the separations from those subsections. The top plot in Fig. 4 is produced by using the lower frequency section of the available spectrum and the bottom plot by using the central section. The central frequency have effect on the range profile created using matched filter also. Even though the performance of BSCT is degraded the tendency of better spacing resolution compared to MF is preserved.

\section{CONCLUSION}

In this paper, the output of a bio-inspired baseband receiver named the BSCT has been studied at radio frequencies. We have observed that the bio-inspired model is capable to resolve fine target details by producing a spacing profile, i.e a profile who is a function of the distance between scatterers, rather than by a typical echo range profile.

Results have shown that the two target range resolution of the BSCT was nearly double that achieved by pulse compression. The strategy which has been used replaces the twotarget resolution problem by the 'single target' problem of just estimating the position of a single peak that indicates the separation between the two targets. This means that the techniques used to estimate the parameters of a single signal can also be brought to bear to further increase the resolution, analogous to measuring the range to a single target, although demonstrating this with RF signals remains a subject for further work.

Future work should investigate how additional parametric frequency estimation techniques, such as MUSIC or Non-linear Least Square curve fitting [22], can be considered to improve the BSCT.

Another subject for further work is to extend this technique to the case where more than two targets are present. Low and high pass filtering of the frequency profile can be applied for handling specific cases as unwanted clutter and worth further exploration together with other standard signal processing techniques. 

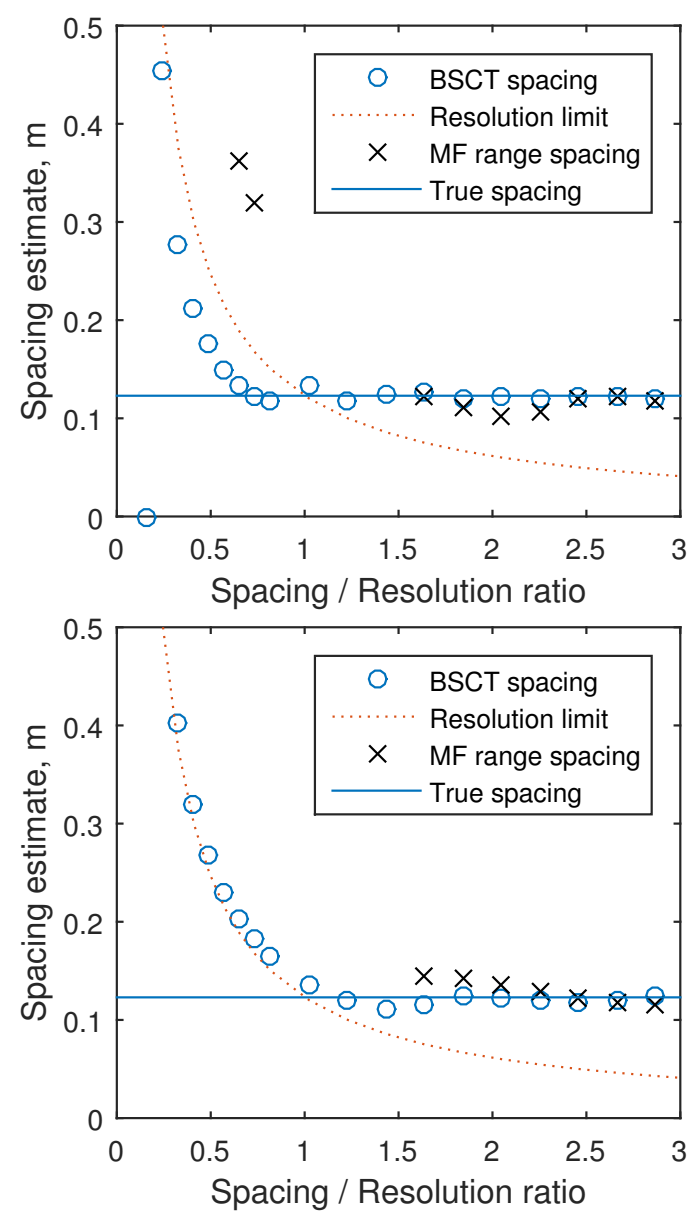

Fig. 4. BSCT spacing estimates. True target separation is $12.2 \mathrm{~cm}$ (shown as continuous horizontal line). The classical resolution limit (5) is shown as dotted line. Plots are produced at different central frequencies

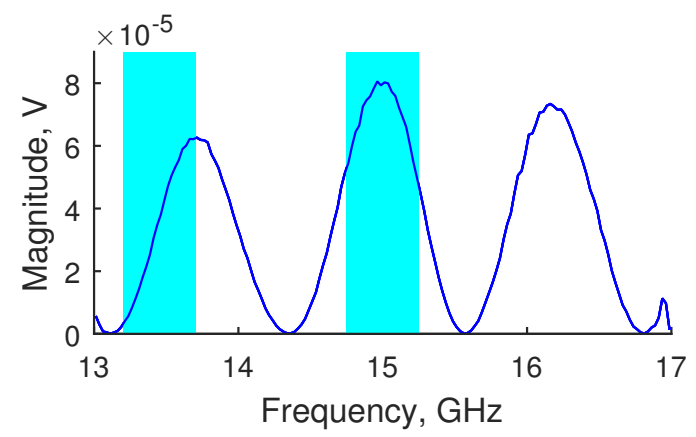

Fig. 5. BSCT frequency profile for target spacing $12.2 \mathrm{~cm}$ and signal bandwidth $4 \mathrm{GHz}$. Two different $0.5 \mathrm{GHz}$ sections are marked

\section{REFERENCES}

[1] A. W. Rihaczek, Principles of high-resolution radar. New York: McGraw-Hill, 1969.

[2] D. Blacknell and H. D. Griffiths, Eds., Radar Automatic Target Recognition (ATR) and Non-Cooperative Target Recognition (NCTR). Stevenage, UK: IET, 2013.

[3] G. W. Stimson, H. D. Griffiths, C. J. Baker, and D. Adamy, Stimson's Introduction to Airborne Radar (3rd Edition). Stevenage, UK: IET, 2014.

[4] D. von Helversen, "Object classification by echolocation in nectar feeding bats: size-independent generalization of shape.” Journal of com- parative physiology. A, Neuroethology, sensory, neural, and behavioral physiology, vol. 190, no. 7, pp. 515-21, 72004.

[5] A. Balleri, H. D. Griffiths, C. J. Baker, K. Woodbridge, and M. W. Holderied, "Analysis of acoustic echoes from a bat-pollinated plant species: Insight into strategies for radar and sonar target classification," IET Radar, Sonar and Navigation, vol. 6, no. 6, pp. 536-544, 2012.

[6] J. A. Simmons, M. Ferragamo, C. F. Moss, S. B. Stevenson, and R. A. Altes, "Discrimination of jittered sonar echoes by the echolocating bat, eptesicus fuscus: The shape of target images in echolocation," Journal of Comparative Physiology A, vol. 167, no. 5, pp. 589-616, 111990.

[7] J. A. Simmons, P. A. Saillant, J. M. Wotton, T. Haresign, M. J. Ferragamo, and C. F. Moss, "Composition of biosonar images for target recognition by echolocating bats," Neural Networks, vol. 8, no. 7-8, pp. 1239-1261, 1995.

[8] S. Schmidt, "Perception of structured phantom targets in the echolocating bat, megadermalyra," The Journal of the Acoustical Society of America, vol. 91, no. 4, pp. 2203-2223, 1992.

[9] R. Simon, M. Knörnschild, M. Tschapka, A. Schneider, N. Passauer, E. K. V. Kalko, and O. von Helversen, "Biosonar resolving power: echoacoustic perception of surface structures in the submillimeter range." Frontiers in physiology, vol. 5, p. 64, 12014.

[10] P. A. Saillant, J. A. Simmons, S. P. Dear, and T. A. McMullen, "A computational model of echo processing and acoustic imaging in frequency-modulated echolocating bats: The spectrogram correlation and transformation receiver," The Journal of the Acoustical Society of America, vol. 94, no. 5, pp. 2691-2712, 111993.

[11] L. Wiegrebe, "An autocorrelation model of bat sonar," Biological cybernetics, vol. 98 , no. 6, pp. 587-595, 2008.

[12] I. Matsuo, K. Kunugiyama, and M. Yano, "An echolocation model for range discrimination of multiple closely spaced objects: transformation of spectrogram into the reflected intensity distribution," The Journal of the Acoustical Society of America, vol. 115, no. 2, pp. 920-928, 22004.

[13] I. Matsuo, "Echolocation of static and moving objects in twodimensional space using bat-like frequency-modulation sound," Frontiers in Physiology, vol. 4, no. July, p. 149, 2013.

[14] N. Neretti, M. I. Sanderson, N. Intrator, and J. A. Simmons, "Timefrequency model for echo-delay resolution in wideband biosonar," The Journal of the Acoustical Society of America, vol. 113, no. 4, pp. 21372145, 2003.

[15] F. Devaud, G. Hayward, and J. J. Soraghan, "P1g-3 evaluation of a bio-inspired range finding algorithm (BIRA)," in IEEE Ultrasonics Symposium, 2006, vol. 1, 2006, pp. 1381-1384.

[16] M. E. Bates and J. A. Simmons, "Perception of echo delay is disrupted by small temporal misalignment of echo harmonics in bat sonar," The Journal of experimental biology, vol. 214, no. 3, pp. 394-401, 2011.

[17] J. A. Simmons and J. E. Gaudette, "Biosonar echo processing by frequency-modulated bats," IET Radar, Sonar and Navigation, vol. 6, no. 6, pp. 556-565, 2012.

[18] M. Park and R. Allen, "Pattern-matching analysis of fine echo delays by the spectrogram correlation and transformation receiver," The Journal of the Acoustical Society of America, vol. 128, no. 3, pp. 1490-1500, 2010.

[19] H. Peremans and J. Hallam, "The spectrogram correlation and transformation receiver, revisited," The Journal of the Acoustical Society of America, vol. 104, no. 2, pp. 1101-1110, 1998.

[20] K. Georgiev, A. Balleri, A. Stove, and M. Holderied, "Baseband version of the bat-inspired spectrogram correlation and transformation receiver," in 2016 IEEE Radar Conference, Philadelphia, USA, May 2016.

[21] K. Georgiev, A. Balleri, A. Stove, and M. W. Holderied, "Enhanced range resolution: comparison with the matched filter," in BiologicallyInspired Radar and Sonar: Lessons from Nature, A. Balleri, H. Griffiths, and C. Baker, Eds. Stevenage, UK: IET, 2017, ch. 3.

[22] P. Stoica and R. Moses, Spectral Analysis of Signals. Pearson Prentice Hall, 2005. 